\title{
Research on the determining method for the relationship of armament combat capability simulation model base on SEM
}

\author{
HE Xin-hua, LU Wan-lin, QU Qiang, LIU Juan \\ Department of Information Engineering, Academy of Armored Forces Engineering, Beijing 100072 , \\ china \\ email:hxh717495@sina.com
}

\begin{abstract}
Keywords: armament system combat capability; simulation model; relationships; SEM
Abstract. It was the base of the model which accurately determined the relationship of each part of the armament system combat capability simulation model. In this paper, structural equation modeling method (SEM) was used to determine relationships between the various components of the armament combat capability simulation model. The specific application of SEM processes and procedures were discussed in detail. Thereby the foundation for establishing the simulation model was established accurately.
\end{abstract}

\section{Introduction}

Between the various components of the combat capability of the unit there is a complex relationship. It's the root causes of combat capability showing whole emergence and non-linear. Therefore, the combat capability of units for an accurate assessment of the relationship between becoming combat capability simulation has become a priority. Traditional combat capability unit based primarily on expert assessment that such an approach is time-consuming and there is a huge subjectivity. So there are a need for a new kind of more scientific and objective method for determining relationships to make up for the shortcomings of traditional methods.

\section{Solution of structural equation model method}

Structural Equation Modeling(SEM) is a field of applied statistics method for analyzing multivariate data affiliated [1-2].It is a comprehensive of many statistical methods, including multiple regression, factor analysis and ideological path analysis ,etc[3-4].

Most applications of statistical methods, the solution of the model it is mainly start from the perspective of the residual sample observations and the actual value. General definitions, $e_{i}=Y_{i}-Y_{i}, e_{i}$ is the difference between the observed values and the estimated, generally is to solve the minimum value of $e_{i}$.

Solving structural equation model using the same method described above thought, as concrete steps: The estimation covariance matrix is derived by using the structure of RAM, make the difference between the sample covariance matrix and the sample covariance matrix is the least. If the model is correct, then the estimated covariance matrix has the following relations with the sample covariance matrix:

$$
\Sigma=\Sigma(\theta) . \Sigma \text { represent sample covariance matrix, }
$$


$\Sigma(\theta)$ represent estimated covariance matrix, two matrices are equal, means the corresponding position elements are equal. Then the parameter matrix $\theta$ to be estimated is solved. From the above equation can be further deduced:

$$
\Sigma(\theta)=E\left[\begin{array}{ll}
Y Y^{T} & Y X^{T} \\
X Y^{T} & X X^{T}
\end{array}\right]=E\left[\begin{array}{ll}
\left(\Lambda_{Y} \eta+\varepsilon\right)\left(\Lambda_{Y} \eta+\varepsilon\right)^{T} & \left(\Lambda_{Y} \eta+\varepsilon\right)\left(\Lambda_{X} \xi+\delta\right)^{T} \\
\left(\Lambda_{X} \xi+\delta\right)\left(\Lambda_{Y} \eta+\varepsilon\right)^{T} & \left(\Lambda_{X} \xi+\delta\right)\left(\Lambda_{X} \xi+\delta\right)^{T}
\end{array}\right]
$$

$$
E\left(X X^{\prime}\right)=\Lambda_{X} E\left(\xi \xi^{\prime}\right) \Lambda_{X}^{\prime}+\Lambda_{X} E\left(\xi \delta^{\prime}\right)+E(\delta \xi) \Lambda_{X}^{\prime}+E\left(\delta \delta^{\prime}\right) \quad \text {,because } \quad \xi \quad \text { is unrelated with }
$$

$\delta$,so $E\left(\xi \delta^{\prime}\right)=0$,note $E\left(\xi \xi^{\prime}\right)=\Phi E\left(\delta \delta^{\prime}\right)=\Theta_{\delta}$,so $\Sigma=\Lambda_{X} \Phi \Lambda_{X}{ }^{T}+\Theta_{\delta}$.So covariance matrix $X$ contains $\Lambda_{X}, \Phi$, and ${ }^{\Theta_{\delta}}$.Same, covariance matrix $Y$ contains $\Lambda_{Y}, \Psi$, and $\Theta_{\varepsilon}$. After solving the estimate covariance and sample covariance, the main idea of model estimation is to find a set of parameters through an iterative approach, make the minimum difference between the sample covariance and the estimated covariance is derived from the model structure.

\section{Method of solving the relationship based on SEM}

Based on the above solving ideological, the solving process of the relationship among the components of weapon equipment operational capability based on the SEM can be divided into the following steps:

(1) Data acquisition and processing SEM is a kind of applied statistics method, the premise is to use a large number of collected statistical samples. Structural equation modeling method on the accuracy terms of sample data requirements are relatively relaxed, allowing presence of sample error. In general, the sample size should be greater than 300, the best case is the sample size is of unknown parameters 5 to 10 times.

(2) Solution of correlation On solution of correlation it can be divided into the two steps: the construction and identification of the model of association relationship and the estimation of the model of correlation. Specific approach is: the probability density function of multivariate normal distribution:

$$
f(\mathrm{Z} ; \Sigma)=\frac{1}{(2 \pi)^{k / 2}|\Sigma|^{1 / 2}} \exp \left[-\frac{Z^{\prime} \Sigma^{-1} Z}{2}\right]
$$

Among them, $\Sigma_{\text {is }}$ the overall covariance matrix, and the number of endogenous indicator variables $X$ and exogenous indicator variables $Y$ are $p$ and $q$, and $\mathrm{Z}_{\text {is a vector of }}(p+q) \times 1$.

Wherein $S$ is the sample covariance matrix.

$$
\begin{aligned}
& \ln L(\theta)=-\frac{n}{2} \ln |\Sigma(\theta)|-\frac{n}{2} \operatorname{tr}\left[\Sigma^{-1}(\theta) S\right]=-\frac{n}{2}\left\{\ln |\Sigma(\theta)|+\operatorname{tr}\left[\Sigma^{-1}(\theta) S\right]\right\} \\
& \ln L(\theta)=-\frac{n}{2} \ln |\Sigma(\theta)|-\frac{n}{2} \operatorname{tr}\left[\Sigma^{-1}(\theta) S\right]=-\frac{n}{2}\left\{\ln |\Sigma(\theta)|+\operatorname{tr}\left[\Sigma^{-1}(\theta) S\right]\right\}
\end{aligned}
$$


(3) Evaluation of the association relation model.

(4) Correction of the association relation model. In the results of the model relationship, characterizing relationship there between with the path between units, a single arrow indicates the role of the relationship, the double arrow characterized association, positive values indicate a positive correlation, a negative value indicates a negative correlation. After a comprehensive evaluation of the evaluation, modify those parts does not meet actual situation. Overall, the model modification aiming at the following general situations: absolute fit indicators $x^{2}$ value is too large.and the expression of the relationship between the units is not correct.

Residual relationship refers to the existence of a path between the elements, that is, the existence of theoretical correlation. But its value is very small (generally thought threshold \pm 0.01 ) is negligible, and the correlation between such units should be deleted.

\section{Instance Analysis}

Described above, the composition of the combat capability of the relationship between units determination method for the practical application of this method, typically by structural equation modeling method based on the idea of design data processing software for data processing. Mainstream application software generally have LISREL and Amos. This paper will based Amos as a platform determined the relationship of the combat capability of the tactical information distribution capabilities, network coverage and network adaptability, showing the specific application of the above theory. Divided into the following steps:

(1) Establishment of combat capability index system. Establish Tactical information distribution capacity, Network coverage and Network adaptability index system. As shown in Figure 1.

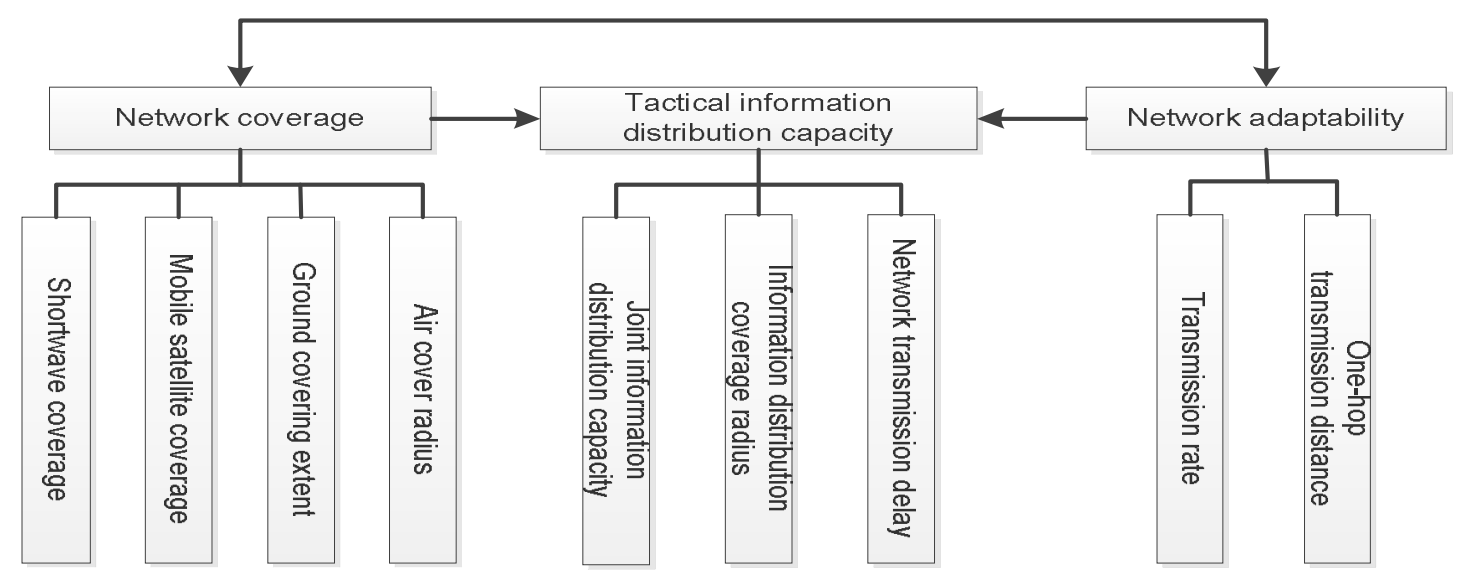

Fig. 1. Combat capability index system.

(2) Relationship building and identification of the model. Establish SEM of Tactical information distribution capacity, Network coverage and Network adaptability. As shown in Figure 2. 


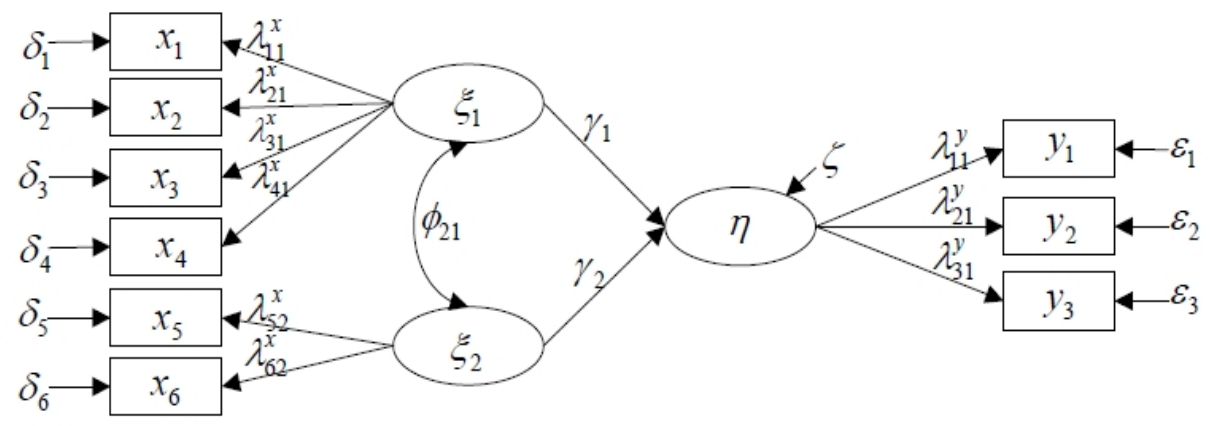

Fig. 2. SEM of Tactical information distribution capacity.

(3) Simulation data of combat ability. In this unit the Army digitization equipment simulation test center, using the Monte Carlo simulation method under different scenarios, tactical information distribution capabilities, network coverage.

(4)The results of parameter estimation. Input the covariance matrix of the simulation data of each scheme and the SEM of Tactical information distribution capacity to the Amos, using the maximum likelihood method to estimate the parameters, obtained the estimated value of the road map and the parameters of the model. According to the estimated value of SEM parameters, get the model of the relationship between Tactical information distribution capacity, Network coverage and Network adaptability is :

$$
\begin{gathered}
\xi_{1}=3.571 x_{1}+3.125 x_{2}+1.667 x_{3}+1.667 x_{2}-1.06 \\
\xi_{2}=3.846 x_{5}+6.25 x_{6}-0.9375 \\
\eta=0.78 \xi_{1}+0.36 \xi_{2}+0.02
\end{gathered}
$$

Through expert assessment and force research, this model of the relationship between Tactical information distribution capacity, Network coverage and Network adaptability in line with the actual situation forces drills.

\section{Conclusion}

It was the key to establish a reasonable simulation model which accurately determined the relationship of each part of the armament system combat capability. This article shows the specific application of SEM processes and procedures. Instance Analysis shows that the value of this method, it has some reference effect.

\section{References}

[1] Wu B.F. A preliminary study on the structural equation [D]. Tianjin: Tianjin University, 2006.

[2] Gelman, A. Inference and monitoring convergence. In W. R. Gilks, S. Richardson and D. J. Spiegelhalter (eds), Markov Chain Monte Carlo in Practice[M]. London: Chapman and Hall, 1996.

[3] Cheng X. Research on the influencing factors of the direct supply mode of electronic retailers [D]. Tianjin: Nankai University, 2010.

[4] Kou P. Company Growth Analysis Based on Nonlinear SEM [D]. Kunming: Kunming University of Science, 2013. 